\title{
Designing universal primers for the isolation of DNA sequences encoding Proanthocyanidins biosynthetic enzymes in Crataegus aronia
}

\author{
Afnan Said Zuiter ${ }^{1}$, Jammal Sawwan ${ }^{1}$ and Ayed Al Abdallat ${ }^{1,2^{*}}$
}

\begin{abstract}
Background: Hawthorn is the common name of all plant species in the genus Crataegus, which belongs to the Rosaceae family. Crataegus are considered useful medicinal plants because of their high content of proanthocyanidins (PAs) and other related compounds. To improve PAs production in Crataegus tissues, the sequences of genes encoding PAs biosynthetic enzymes are required.

Findings: Different bioinformatics tools, including BLAST, multiple sequence alignment and alignment PCR analysis were used to design primers suitable for the amplification of DNA fragments from 10 candidate genes encoding enzymes involved in PAs biosynthesis in C. aronia. DNA sequencing results proved the utility of the designed primers. The primers were used successfully to amplify DNA fragments of different PAs biosynthesis genes in different Rosaceae plants.
\end{abstract}

Conclusion: To the best of our knowledge, this is the first use of the alignment PCR approach to isolate DNA sequences encoding PAs biosynthetic enzymes in Rosaceae plants.

Keywords: Alignment PCR Analysis, BLAST, Multiple sequence alignment, Proanthocyanidins, Rosaceae

\section{Background}

Hawthorn (Crataegus spp.) is a member of the Rosaceae family, belonging to the Spiraeoideae subfamily in the Pyroidae supertribe in the Pyreae subtribe [1]. There are approximately 280 species in the genus Crataegus, which are widely distributed across the Mediterranean, North Africa, Europe, Central and Eastern Asia and North America [2]. In the eastern Mediterranean region, the predominant Crataegus species is C. aronia L., which is found on dry hillsides, mountains and in areas receiving more than $300 \mathrm{~mm}$ annual rainfall [3].

In folk medicine, a decoction of Crataegus leaves and unripe fruits has been used to treat disorders such as cardiovascular diseases, cancer, diabetes, and impotence $[2,4]$. The medicinal properties of Crataegus are primarily related to its antioxidant activities and the reduction of free radical-induced oxidative stress $[5,6]$, mediated by

\footnotetext{
* Correspondence: a.alabdallat@ju.edu.jo

'Department of Horticulture and Crop Science, Faculty of Agriculture, University of Jordan, Amman 11942, Jordan

${ }^{2}$ Hamdi Mango Center for Scientific Research, University of Jordan, Amman 11942, Jordan
}

secondary metabolites, such as flavonoids, oligomeric Proanthocyanidins (PAs), ethanobotanical and ethanopharmacological compounds [7].

PAs, also known as condensed tannins, are polyphenolic compounds formed as a branch of the flavonoid biosynthetic pathway [8]. PAs are found in the fruits, bark, leaves and seeds of many plants including Crataegus $[2,9]$ and their function include defense against herbivores and resistance to abiotic stresses [9]. They are also important quality components of many fruits, providing the flavor and color to beverages [10]. The PAs biosynthesis pathway and its regulatory genes have been dissected genetically and biochemically in a number of plants [11] including Arabidopsis [12,13] Vitis vinifera $[10,14,15]$, bilberry (Vaccinium myrtillus) [16], soybean (Glycine max) [17] and persimmon (Diospyros kaki) [18], but not in Crataegus. Therefore, there is a need to identify DNA sequences encoding phenylpropanoid biosynthesis enzymes in Crataegus. In this study, bioinformatics tools were used to design specific primers to amplify DNA sequences of key genes encoding enzymes involved in the PAs biosynthetic pathway in C. aronia. 


\section{Findings}

\section{Designing of Primers for the Isolation of PAs Biosynthetic genes}

First, DNA sequences encoding PAs biosynthetic enzymes from four different Pyreae subtribe genera were retrieved from the NCBI databases, which resulted in several DNA sequences representing ten selected PAs biosynthesis enzymes (Additional file 1: Table S1). All the DNA sequences were complete coding regions, except for the 4- $\mathrm{Cl}$ gene. BLAST searching with the selected DNA sequences retrieved related DNA sequences from different plant, including DNA sequences encoding PAs biosynthetic enzymes from the Spiraeoideae, Rosaceae, Rosales and Fabids taxa (data not shown). All retrieved sequences were analyzed for their nucleic acid type (genomic DNA or mRNA or EST), the presence or absence of introns, their length (partial or full length) and for their redundancy in different nucleotide databases.

Overlapping partial DNA sequences were gathered and assembled into contigs using the VectorNTI software. Finally, the DNA sequences of each PAs biosynthetic gene were grouped according to their taxon and then used to design universal primers using alignment PCR analysis in the VectorNTI software.

To design of PAs biosynthesis genes specific primers, multiple sequence alignment (MSA) analysis was performed initially among all retrieved DNA sequences from selected taxa using ClustalW in the Vector NTI suite. The MSA results were inspected and filtered to exclude DNA sequences with low levels of similarity and wide gaps. Secondary and tertiary MSAs were performed on the most similar DNA sequences until the aligned sequences showed large areas of sequence similarity. Additional file 2 shows the ANS genes' alignment as an example. In general, conserved regions in the DNA sequences of PAs biosynthetic enzymes were identified in groups of closely related genera in the Rosaceae family, with some exceptions (data not shown). Alignment PCR analysis was then performed and the most suitable primers for PCR amplification of targeted genes in Rosaceae plants were selected and subjected to further analysis (Table 1).

To validate the alignment PCR results, the primers were BLAST searched against the nucleotide databases of the Genome Database for Rosaceae (http://www.rosaceae.org) and against the NCBI GenBank nucleotide databases of flowering plants. As expected, the percentage of similarity between the designed primers and the DNA sequences of the targeted PAs genes in the Rosaceae family was very high, with minor exceptions for Malus, Prunus and Fragaria spp. (see Additional file 3). In addition, many of the primers were conserved in different flowering plants, including grape, soybean, arabidopsis and poplar (data not shown).
Table 1 Characteristics of the designed primers specific for Rosaceae PAs biosynthesis genes used in this study

\begin{tabular}{|c|c|c|c|}
\hline $\begin{array}{l}\text { Gene } \\
\text { name }\end{array}$ & Primer & Primer sequence $\left(5^{\prime} \rightarrow 3^{\prime}\right)$ & $\mathrm{T}_{\mathrm{m}}{ }^{\circ} \mathrm{C}^{\mathrm{a}}$ \\
\hline \multirow[t]{4}{*}{$\overline{A N R}$} & ANRFwd1 & CCCACCTMACAGCACTACAA ${ }^{b}$ & 53.8 \\
\hline & ANRRev1 & TACCGACCAGAAGCAGATTC & 51.8 \\
\hline & ANRFwd2 & GGAGTGATTTGAGTTCTTG & 49.7 \\
\hline & ANRRev2 & GGAAAATCTCCAAACTCAGT & 47.7 \\
\hline \multirow[t]{4}{*}{ ANS } & ANSFwd1 & TTTGAYCTTCCCATTGAGCA & 49.7 \\
\hline & ANSRev1 & GCATITTGGGTAGTAGTTGA & 47.7 \\
\hline & ANSFwd2 & CAGCTTGAGTGGGAGGAYTA & 53.8 \\
\hline & ANSRev2 & GCAGGCCRGGAACCATGTTG & 57.9 \\
\hline \multirow[t]{4}{*}{$4-C L$} & 4-CLFwd1 & CCTGGAGAGATTTGCATCAG & 51.8 \\
\hline & 4-CLRev1 & AATACTCGATTTATTCTTTTATA & 42.8 \\
\hline & 4-CLFwd2 & ATCATGAAAGGTTAYCTTAATG & 45.5 \\
\hline & 4-CLRev2 & GGAATGGCTTCGATGAAAAA & 47.7 \\
\hline \multirow[t]{4}{*}{$\mathrm{CHI}$} & CHIFwd1 & AGGGGGTYGGAGATTCAGGG & 55.9 \\
\hline & CHIRev1 & GGGAAGKTTTGATCYTTGAA & 45.6 \\
\hline & $\mathrm{CHIFwd2}$ & TTCGTGAAGTTCACGGCGAT & 51.8 \\
\hline & CHIRev2 & TGGGAAGGTCTGATCTTTGA & 49.7 \\
\hline \multirow[t]{4}{*}{$\mathrm{C} 4 \mathrm{H}$} & C4HFwd1 & CTCCGYATGGGRCAGCGCAA & 60 \\
\hline & C4HRev1 & TTGTTGTACATCATMAGCTG & 47.7 \\
\hline & C4HFwd2 & CAGCTBATGATGTACAACAA & 47.7 \\
\hline & C4HRev2 & TGGATTTCRGGGTGGTTCAC & 53.8 \\
\hline \multirow[t]{4}{*}{ CHS } & CHSFwd1 & AAGGCCATYAAGGAATGGGG & 53.8 \\
\hline & CHSRev1 & AATGTRAGCCCDACTTCACG & 53.8 \\
\hline & CHSFwd2 & AAGGAATGGGGHCAGCCCAA & 55.9 \\
\hline & CHSRev2 & CTCACYTCRTCCAAAATAAA & 47.7 \\
\hline \multirow[t]{4}{*}{$D F R$} & DFRFwd1 & CCTGACGCTGTGGAAGGCGG & 60 \\
\hline & DFRRev1 & TAATGGTRATGAAATCAATG & 43.6 \\
\hline & DFRFwd2 & TGCASCGGAGTGTTYCATGT & 53.8 \\
\hline & DFRRev2 & TGAGGCTTGGTGGCATRGATGG & 58.6 \\
\hline \multirow[t]{4}{*}{ LAR } & LARFwd1 & GTTCGTMGCYGAAGCCAGCC & 60 \\
\hline & LARRev1 & TGGAACYGATCCAACGGTGG & 55.9 \\
\hline & LARFwd2 & AGGGCYRATCCGGTKGAACC & 60 \\
\hline & LARRev2 & AGGGTSCGRCCAATTTTCTT & 51.8 \\
\hline \multirow[t]{4}{*}{$\mathrm{F} 3 \mathrm{H}$} & F3HFwd1 & GACATGTCCGGYGGCAAAAAGGG & 60.6 \\
\hline & F3HRev1 & СTCATCTTCTTCTTGTACAT & 45.6 \\
\hline & F3HFwd2 & ACGTGGATCACCGTTCAACC & 53.8 \\
\hline & F3HRev2 & GGTTGAACGGTGATCCACGT & 53.8 \\
\hline \multirow[t]{3}{*}{$\overline{P A L}$} & PALFwd1 & ATCGATGTTTCRAGGAACAA & 47.7 \\
\hline & PALRev1 & AAAGAGTTAACATCTTGGTT & 43.6 \\
\hline & PALRev2 & AAAAATGTRGAAGACATGAG & 45.6 \\
\hline
\end{tabular}

a Melting temperature.

${ }^{b}$ Degenerate nucleotides codes where $B(C / G / T), D(A / G / T), H(A / C / T), K(G / T)$, $M(A / C), N(A / C / G / T), R(A / G), S(G / C), V(A / C / G), W(A / T), Y(C / T)$.

However, some low similarity (one or two mismatches) between the designed primers and the DNA sequences of the targeted PAs genes in some Rosaceae family members were observed (data not shown). To resolve this, 
degenerate nucleotides were included in the primer sequences to ensure their functionality with different plants from the Rosaceae family (Table 1).

\section{Testing the designed primers using RT-PCR in different Rosaceae plants}

The efficiency of the designed primers in amplifying DNA fragments of the corresponding PAs biosynthesis genes from $C$. aronia was tested using RT-PCR. In general, DNA fragments of $C$. aronia PAs biosynthesis genes were successfully amplified using cDNA prepared from C. aronia callus cells and different combinations of designed primers (Additional file 4; Figure 1). Successful primer combinations produced PCR amplicons from C. aronia cDNA of the expected size of the corresponding DNA fragments in the target gene.

DNA sequencing of the positive amplicons confirmed the results of the RT-PCR analysis (data not shown). The sequencing data were further analyzed using bioinformatics tools, such as BLAST searching against the

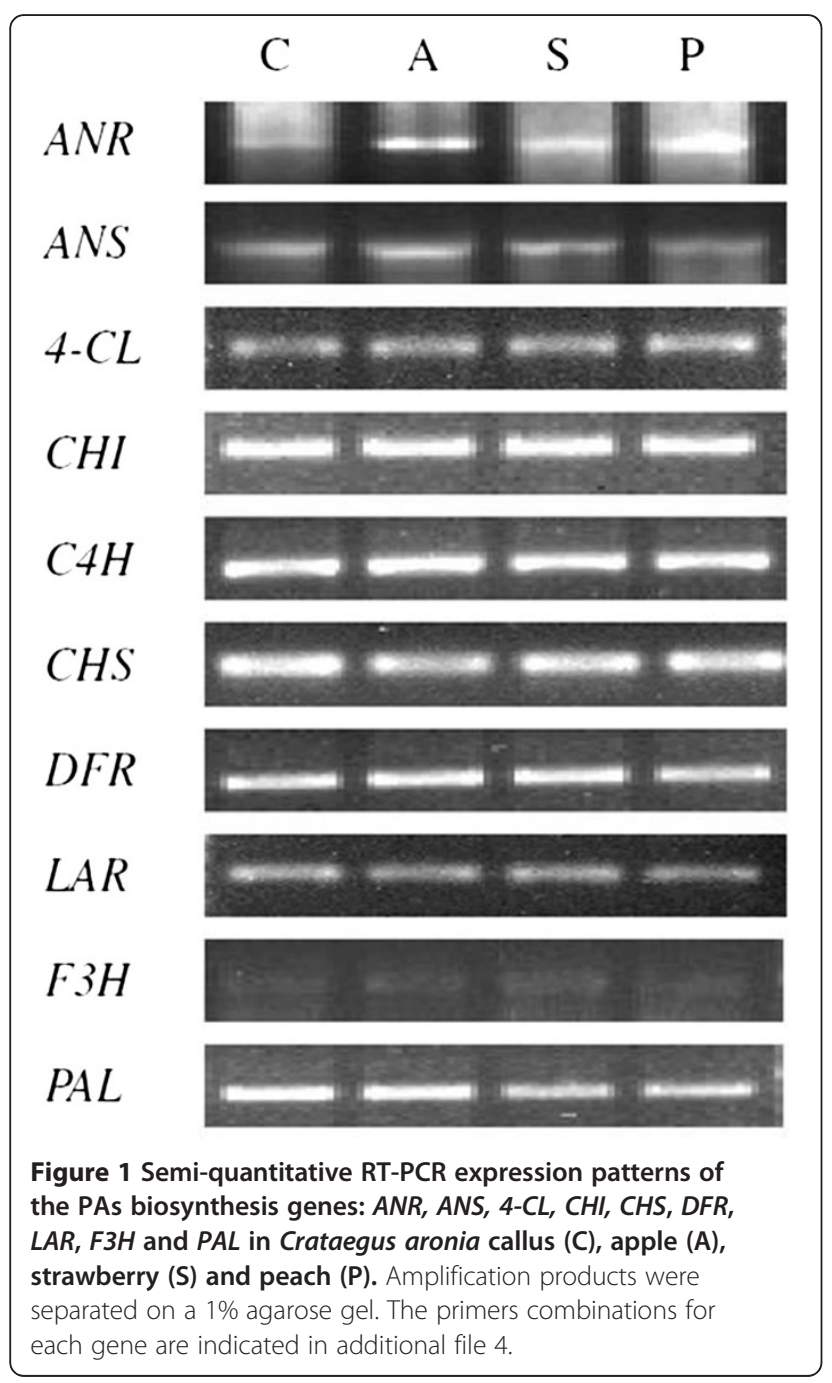

Genome Database for Rosaceae (http://www.rosaceae. org) and the NCBI GenBank nucleotide databases of flowering plants. DNA sequencing results and bioinformatics analysis of the amplicons confirmed that the PCR products were DNA fragments of PAs biosynthesis genes from C. aronia.

To test designed primers' ability to amplify PCR products in different Rosaceae plants, the most successful primer combinations in C. aronia (Additional file 4) were tested using cDNA prepared from leaf samples from apple, strawberry and peach. The designed primers successfully amplified PCR products from cDNA prepared from $C$. aronia callus tissue, apple, strawberry and peach (Figure 1).

\section{Discussion}

To gain better understanding of the PAs biosynthesis pathway and the mechanism governing their synthesis in plants, several genes encoding PAs biosynthetic enzymes have been isolated [10-19]. Metabolic and genetic engineering systems have used this genetic information to boost the production of PAs in different plants [20,21]. In the present study, alignment PCR was used to design functional primers for genes encoding PAs biosynthetic enzymes in Rosaceae plants. Partial DNA sequences were obtained for 10 targeted genes encoding PAs biosynthetic enzymes from $C$. aronia using the designed primers. Many of the designed primers are conserved across different plant species, indicating that they could be used to obtain genes encoding PAs biosynthetic enzymes in nonrelated organism. The conserved primers might be used in colinearity and comparative genomics studies between highly related species [22].

The alignment PCR approach easily, specifically and effectively produced DNA sequences from the targeted genes in different plant species. Degenerate primers, rapid amplification of cDNA ends (RACE) primer technology and PCR-based walking strategies were used to characterize PAs biosynthetic enzyme genes ANR, ANS, $F 3 H, D F R, L A R$ and FS in strawberry [23]. In that study, the degenerate primers were designed using MSA based on protein sequences. The same strategy was used to amplify full-length coding sequences of the $A N R$ and $L A R$ genes from grape [10].

Compared with the alignment PCR analysis, the degenerate primers approach is based on extracting DNA sequences from conserved amino acids in homologous proteins [24]. The degenerate primers are designed to amplify related but not identical DNA sequences. Such an approach might result in lower specificity of the designed primers and increase probability of producing non-targeted amplicons [24,25].

Several public databases have been developed for designing universal primers for a particular gene across 
different taxa, e.g. UniPrime [26], and UniPrime2 [27]. The alignment PCR protocol described in this study adapts the same principles, parameters and approaches for universal primers design described in the public databases. However, such databases are not suited for partial CDS sequences that are difficult to handle in MSA analysis. In this study, several partial DNA sequences retrieved from EST databases were subjected to contig assembly to produce full-length DNA sequences that were easier to analyze using MSA. The contig assembly of partial CDS approach could be used to improve currently existing public universal primers databases.

\section{Conclusions}

In this study, genetic information related to 10 different genes encoding PAs biosynthetic enzymes from $C$. aronia plant were obtained. Such information can be used to clone the full-length gene sequences from $C$. aronia. The information can also be used to improve PAs production in $C$. aronia using genetic engineering and tissue culture systems developed specifically for this plant species [28].

The designed primers showed high levels of sequence similarity with their corresponding genes in different Rosaceae plants; therefore, they could be used to isolate DNA fragments of PAs genes from different Rosaceae plants.

\section{Methods}

\section{Plant material}

Callus cultures of $C$. aronia were established as described previously [28]. To test the functionality of the identify primers with other Rosaceae species, leaf samples from apple (Malus domestica), peach (Prunus persica) and strawberry (Fragaria $x$ ananassa) were used.

\section{Bioinformatics analysis}

First, DNA or protein sequences of the targeted PAs biosynthetic enzymes in Crataegus and closely related plant species and genera were retrieved from NCBI GenBank databases (Additional file 1: Table S1). The sequences were subjected to different BLAST algorithms to gather DNA sequences of the corresponding PA biosynthesis from different plant taxa, especially the Pyreae subtribe, Pyrodae supertribe, Spiraeoideae subfamily, Rosaceae family, Rosales order and Fabids. The obtained BLAST results were filtered and the best hits (expectation value $(\triangle \mathrm{E})$ less than 1e-50) were chosen for alignment PCR analysis. The retrieved DNA sequences were subjected to MSA analysis using the ClustalW program in the Vector $\mathrm{NTI}^{\mathrm{m}}$ Suite version 11.5 (Invitrogen, Carlsbad, CA, USA). The MSA results were used to design taxa specific primers based on conserved DNA sequences, using alignment PCR and 'primer design' tools, as described in the VectorNTI Suite version 11.5 manual.
Finally, the best primers combinations were selected for the PCR analysis.

\section{RNA extraction and reverse transcription $P C R$}

Callus cells of $C$. aronia and leaf samples of different Rosaceae plants were used to extract total RNA using the EZ-10 Spin Column Total RNA Mini-Preps Super Kit (Biobasic Inc., Ontario, Canada), following the manufacturer's instructions. For reverse transcription (RT) -PCR analysis, $1 \mu \mathrm{g}$ of the isolated total RNA samples were converted into first-strand cDNA using the GoScript $^{\mathrm{TM}}$ reverse transcription kit (Promega, Madison, Wisconsin), following the manufacturer's instructions. The synthesized cDNAs were used as PCR templates to amplify DNA fragments encoding PAs biosynthetic enzymes. The PCR was performed in an Applied Biosystems 9700 thermocycler (Applied Biosystems, Carlsbad, CA, USA) using the i-MAXII PCR Master Mix solution (iNtRON Biotechnology, Seoul, Korea) and different primer combinations (Table 1). The $25 \mu \mathrm{l}$ reactions contained $2.5 \mu \mathrm{l}$ of the synthesized cDNA, $0.5 \mu \mathrm{M}$ of each primer and $12.5 \mu \mathrm{l}$ of i-MAXII solution, following the manufacturer's instructions. The PCR conditions were $94^{\circ} \mathrm{C}$ for $5 \mathrm{~min}$; 35 cycles of $94^{\circ} \mathrm{C}$ for $45 \mathrm{sec}-$ onds, $50-60^{\circ} \mathrm{C}$ (depending on the primer combination) for 30 seconds, and $72^{\circ} \mathrm{C}$ for 1 minute; and a final extension at $72^{\circ} \mathrm{C}$ for 10 minutes. The PCR products were separated on $1.5 \%$ agarose gels and visualized using the Safe Red stain (iNtRON Biotechnology, Seoul, Korea) using Gel $\operatorname{Doc}^{\text {Tix }} \mathrm{XR}+($ BioRad, Hercules, CA). DNA fragments of the expected sizes were excised from the agarose gel and extracted using the Wizard ${ }^{\circledR}$ SV Gel and PCR Cleanup System (Promega, Madison, Wisconsin), following the manufacturer's instructions. The DNA fragments were sequenced using an ABI 3730XL DNA sequencer by Macrogen Inc. (Seoul, Korea).

\section{Additional files}

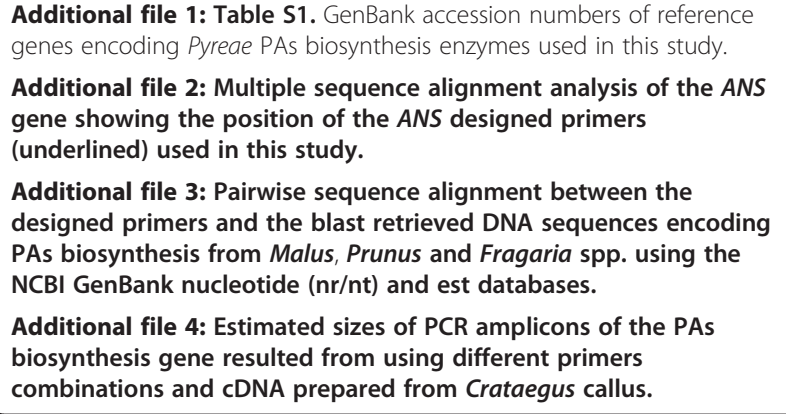

Additional file 1: Table S1. GenBank accession numbers of reference genes encoding Pyreae PAs biosynthesis enzymes used in this study.

Additional file 2: Multiple sequence alignment analysis of the ANS gene showing the position of the ANS designed primers (underlined) used in this study.

Additional file 3: Pairwise sequence alignment between the designed primers and the blast retrieved DNA sequences encoding PAs biosynthesis from Malus, Prunus and Fragaria spp. using the NCBI GenBank nucleotide (nr/nt) and est databases.

Additional file 4: Estimated sizes of PCR amplicons of the PAs biosynthesis gene resulted from using different primers combinations and cDNA prepared from Crataegus callus.

\section{Abbreviations}

ANR: Anthocyanidin reductase; ANS: Anthocyanidin synthase; C4H: Cinnamate 4-hydroxylase; CHI: Chalcone isomerase; CHS: Chalcone synthase; 4-CL: 4-Coumarate:Coenzyme A ligase; DFR: Dihydroflavonol reductase; F3H: Flavonoid 3-hydroxylase; LAR: Leucoanthocyanidin reductase; 
MSA: Multiple sequence alignment; PAL: Phenylalanine ammonia lyase; PAs: Proanthocyanidins.

\section{Competing interests}

The authors declare that they have no competing interests.

\section{Authors' contributions}

ASZ performed most of the experimental work and bioinformatics analysis; JSS developed the conceptual aspects of the work and edited the manuscript; AMA conceived the research, helped in the molecular and bioinformatics work and drafted the manuscript; all authors read and approved the final manuscript.

\section{Acknowledgments}

We gratefully acknowledge Mrs. Samar Misbeh for technical assistance. This work was supported in part by a grant from the Hamdi Mango Center for Scientific Research, University of Jordan, and in part by a grant from the Deanship of Scientific Research, University of Jordan.

Received: 10 May 2012 Accepted: 2 August 2012

Published: 10 August 2012

\section{References}

1. Potter D, Eriksson T, Evans R, Oh S, Smedmark J, Morgan D, Kerr M, Robertson K, Arsenault M, Dickinson T, Campbell C: Phylogeny and classification of Rosaceae. Plant Systematics and Evolution 2007, 266:5-43.

2. Chang Q, Zuo Z, Harrison F, Chow M: Hawthorn. J Clin Pharmacol 2002, 42:605-612.

3. Khalil R, Abuharfeil N, Shabsoug B: The effect of Crataegus aronia aqueous extract in rabbits fed with high cholesterol diet. Eur J Sci Res 2008, 22:352-360.

4. Ljubuncic P, Portnaya I, Cogan U, Azaizeh H, Bomzon A: Antioxidant activity of Crataegus aronia aqueous extract used in traditional Arab medicine in Israel. J Ethnopharmacol 2005, 101:153-161.

5. Bahorun T, Aumjaud E, Ramphul H, Rycha M, Luximon-Ramma A, Trotin F, Aruoma O: Phenolic constituents and antioxidant capacities of Crataegus monogyna (Hawthorn) callus extracts. Nahrung 2003, 47:191-198.

6. Sokol-Letowska A, Oszmianski J, Wojdylo A: Antioxidant activity of the phenolic compounds of hawthorn, pine and skullcap. Food Chem 2007, 103:853-859.

7. Froehlicher T, Hennebelle T, Martin-Nizard F, Cleenewerck P, Hilbert J L Trotin F, Grec S: Phenolic profiles and antioxidative effects of hawthorn cell suspensions, fresh fruits, and medicinal dried parts. Food Chem 2009, 115:897-903.

8. Davies KM, Schwinn KE: Molecular biology and biotechnology of flavonoid biosynthesis. In Flavonoids: Chemistry, Biochemistry and Applications. Edited by Andersen OM, Markham KR. Florida: CRC Press; 2006:144-201.

9. Dixon $R$, Xie DY, Sharma S: Proanthocyanidins- a final frontier in flavonoid research? New Phytol 2005, 165:9-28.

10. Bogs J, Downey M, Harvey J, Ashton A, Tanner G, Robinson S: Proanthocyanidin synthesis and expression of genes encoding leucoanthocyanidin reductase and anthocyanidin reductase in developing grape berries and grapevine leaves. Plant Physiol 2005 139:652-663.

11. He F, Pan QH, Shi Y, Duan CQ: Biosynthesis and genetic regulation of proanthocyanidins in plants. Molecules 2008, 13:2674-2703.

12. Abrahams S, Tanner G, Larkin P, Ashton A: Identification and biochemical characterization of mutants in the proanthocyanidin pathway in Arabidopsis. Plant Physiol 2002, 130:561-576.

13. Owens D, Alerding A, Crosby K, Bandara A, Westwood J, Winkel B: Functional analysis of a predicted flavonol synthase gene family in Arabidopsis. Plant Physiol 2008, 147:1046-1061.

14. Fujita A, Soma N, Goto-Yamamoto N, Mizuno A, Kiso K, Hashizume K: Effect of shading on proanthocyanidin biosynthesis in the grape berry. Journal of the Japanese Society for Horticultural Science 2007, 76:112-119.

15. Terrier N, Torregrosa L, Ageorges A, Vialet S, Verries C, Cheynier V, Romieu C: Ectopic expression of VvMybPA2 promotes proanthocyanidins biosynthesis in grapevine and suggests additional targets in the pathway. Plant Physiol 2009, 149:1028-1041.

16. Jaakola L, Maatta K, Pirttila A, Torronen R, Karenlampi S, Hohtola A: Expression of genes involved in anthocyanin biosynthesis in relation to anthocyanin, proanthocyanidin, and flavonol levels during bilberry fruit development. Plant Physiol 2002, 130:729-739.

17. Todd J, Vokin L: Pigmented soybean (Glycine max) seed coats accumulate proanthocyanidin during development. Plant Physiol 1993, 102:663-670.

18. Ikegami A, Eguchi S, Kitajima A, Inoue K, Yonemori K: Identification of genes involved in proanthocyanidin biosynthesis of persimmon (Diospyros kaki) fruit. Plant Sci 2007, 172:1037-1047.

19. Paolocci F, Bovone T, Tosti N, Arcioni S, Damiani F: Light and an exogenous transcription factor qualitatively and quantitatively affect the biosynthetic pathway of condensed tannins in Lotus corniculatus leaves. J Exp Bot 2005, 56:1093-1103.

20. Boudet AM: Evolution and current status of research in phenolic compounds. Phytochemistry 2007, 68:2722-2735.

21. Chemler J, Leonard E, Koffas M: Flavonoid biotransformation in microorganisms. In Anthocyanins: Biosynthesis, Functions, and Applications. Edited by Gould K, Davies K, Winefield C. New York: Springer Science and Business media; 2009:191-238.

22. Bossolini E, Krattinger S, Keller B: Development of simple sequence repeat markers specific for the Lr34 resistance region of wheat using sequence information from rice and Aegilops tauschii. Theor Appl Genet 2006, 113(6):1049-1062.

23. Almedia J, D'Amico E, Preuss A, Carbone F, de vos Rec CH, Deiml B, Mourgues F, Perrotta G, Fischer T, Bovy A, Martens S, Rosati C: Characterization of major enzymes and genes involved in flavonoid and proanthocyanidin biosynthesis during fruit development in strawberry (Fragaria x ananassa). Arch Biochem Biophys 2007, 465:61-71.

24. Wei $X$, Kuhn D, Narasimhan G: Degenerate primer design via clustering In Proc. 2nd IEEE Computer Society Bioinformatics Conference (CSB). 83: 83; 2003:75-83.

25. Linhart C, Shamir R: The degenerate primer design problem: theory and applications. J Comput Biol 2005, 12:431-456.

26. Bekaert $M$, Teeling M: UniPrime: a workflow-based platform for improved universal primer design. Nucleic Acids Res 2008, 36:e56.

27. Boutros R, Stokes N, Bekaert M, Teeling E: UniPrime2: a web service providing easier Universal Primer design. Nucleic Acids Res 2009, 37:209-213.

28. Al Abdallat A, Sawwan J, Al Zoubi B: Agrobacterium tumefaciens-mediated transformation of callus cells of Crataegus aronia. Plant Cell Tissue and Organ Culture 2010, 104:31-39.

doi:10.1186/1756-0500-5-427

Cite this article as: Zuiter et al.: Designing universal primers for the isolation of DNA sequences encoding Proanthocyanidins biosynthetic enzymes in Crataegus aronia. BMC Research Notes 2012 5:427.

\section{Submit your next manuscript to BioMed Central and take full advantage of:}

- Convenient online submission

- Thorough peer review

- No space constraints or color figure charges

- Immediate publication on acceptance

- Inclusion in PubMed, CAS, Scopus and Google Scholar

- Research which is freely available for redistribution 